\title{
Linear and Nonlinear Terrain Deformation Maps From a Reduced Set of Interferometric SAR Images
}

\author{
Oscar Mora, Member, IEEE, Jordi J. Mallorqui, Member, IEEE, and Antoni Broquetas, Member, IEEE
}

\begin{abstract}
In this paper, an advanced technique for the generation of deformation maps using synthetic aperture radar (SAR) data is presented. The algorithm estimates the linear and nonlinear components of the displacement, the error of the digital elevation model (DEM) used to cancel the topographic terms, and the atmospheric artifacts from a reduced set of low spatial resolution interferograms. The pixel candidates are selected from those presenting a good coherence level in the whole set of interferograms and the resulting nonuniform mesh tessellated with the Delauney triangulation to establish connections among them. The linear component of movement and DEM error are estimated adjusting a linear model to the data only on the connections. Later on, this information, once unwrapped to retrieve the absolute values, is used to calculate the nonlinear component of movement and atmospheric artifacts with alternate filtering techniques in both temporal and spatial domains. The method presents high flexibility with respect the required number of images and the baselines length. However, better results are obtained with large datasets of short baseline interferograms. The technique has been tested with European Remote Sensing SAR data from an area of Catalonia (Spain) and validated with on-field precise leveling measurements.
\end{abstract}

Index Terms-Differential interferometry (DInSAR), digital elevation model (DEM), nonlinear movement, subsidence, synthetic aperture radar (SAR), terrain deformation.

\section{INTRODUCTION}

$\mathbf{T}$ HE DETECTION of earth surface movements using remote sensing techniques has shown excellent results in the last years of research [1]-[17]. The first steps in differential synthetic aperture radar (SAR) interferometry (DInSAR) were made combining a pair of short-baseline SAR images enough separated in time to generate the associated interferogram. If the topographic phase can be neglected, due to the short spatial baseline, in front of the one caused by deformation, or it is removed using an external digital elevation model (DEM), the unwrapped interferometric phase shows the spatial distribution and magnitude of the displacement. This technique was successfully used to monitor single deformation episodes like the ones caused by earthquakes [1], [2] or volcanoes [3], due to the short time gap necessary between both SAR acquisitions,

Manuscript received September 30, 2002; revised May 16, 2003. This work was supported by the European Space Agency (ESA) under the EO Project of Category 1 A03.421 and the Spanish CICYT under projects TIC1999-1050-C03-01 and TIC2002-04451-C02-01.

O. Mora was with the Departament of Teoria del Senyal i Comunicacions (TSC), Universitat Politècnica de Catalunya (UPC). He is now with the Institut Cartogràfic de Catalunya (ICC), 08038 Barcelona, Spain (e-mail: omora@icc.es).

J. J. Mallorqui and A. Broquetas are with the Departament of Teoria del Senyal i Comunicacions (TSC), Universitat Politècnica de Catalunya (UPC), 08034 Barcelona, Spain (e-mail: mallorqui @tsc.upc.es; toni@ tsc.upc.es).

Digital Object Identifier 10.1109/TGRS.2003.814657 which reduces the temporal decorrelation and allows working with a dense grid of pixels. Differential interferometry has also been used to monitor landslides in alpine zones above the tree line, because on surfaces with sparse vegetation and bare soil or rock the coherence is preserved over long periods [4]. Nevertheless, in the most general cases when monitoring low-velocity deformations differential interferograms are forced to have a large temporal baseline and consequently the temporal decorrelation degrades the interferometric phase making almost impossible the extraction of useful information unless large coherent areas are present. An additional limitation, common to both short and large temporal baseline differential interferograms, is the presence of atmospheric artifacts that degrade the quality of the displacement estimation. Note that usual changes in the troposphere from one day to another can produce different time delays in the propagation of the signal resulting in phase patterns similar to the deformation ones. In order to overcome these inherent limitations, various techniques have been published during the last years to study the temporal evolution of deformations from large datasets of images [5]-[17].

The phase gradient approach to stacking interferograms [5] is used to construct averages and differences of interferograms without phase unwrapping, allowing the study of surface change detection by increasing fringe clarity and decreasing the errors introduced by the atmospheric contribution. The method has been applied to study fault creeps for earthquake physics and hazard mitigation [6]. In order to minimize the effects of the DEM inaccuracies and the spatial decorrelation, some techniques work with small baseline interferograms generated from the image dataset, which can cause the presence of different subsets of interferograms with no common images. Initially, only one deformation time series for each subset were obtained [7], [8]. The technique has been extended to the subset problem by means of the singular value decomposition (SVD) method [9], which applies a minimum-norm criterion to the velocity deformation estimation [10]. This technique requires the unwrapping of the interferograms over the sparse-grid formed by the pixels with coherence over a given threshold. The method calculates the time sequence deformation and estimates the DEM error and the atmospheric artifacts present in the interferograms, in a similar way as the Permanent Scatterers technique [13]. A complementary approach, also based on the application of the SVD method to link independent SAR acquisitions datasets, was presented in [11]. Instead of looking for large scale deformations with reduced spatial resolutions, the algorithm was able to monitor localized deformation phenomena at high resolution of highly coherent structures, like buildings. 
The technique of the Permanent Scatterers (PS) also uses large stacks of images to generate the differential interferograms with respect a common master image for each available acquisition, even if the baseline is larger than the critical one and the generated interferogram highly affected by spatial decorrelation [12]. The high dispersion of baseline values and the limited accuracy of the available DEM make impossible the usage of a coherence criterion to select the pixels with good phase quality, since with the largest baselines the topographic component has to be accurately removed from the interferometric phase prior coherence computation. With the PS technique, pixels are selected from the study of its amplitude stability along the whole set of images, which requires a minimum of 30 images and its proper radiometric calibration for a reliable selection; therefore, the maximum resolution of the SAR images is preserved [12]. A linear model is adjusted to the data to estimate the deformation linear velocity and the DEM error for each selected pixel. Finally, the nonlinear component of movement and the atmospheric phase screen for each image is computed with a combined spatio-temporal filtering [13]. Recently, the PS technique has been used to combine nonhomogeneous datasets, not acquired within the same track frame and mode, over the same area [14]. In the preliminary results, 160 descending mode images from two different tracks and 30 ascending mode images were combined increasing the spatial density of radar benchmarks. The PS technique has also been applied on landslides monitoring where correlation is usually low, and the detection of isolated stable scatterers is crucial [15].

The algorithm presented in this paper is also able to retrieve the linear and nonlinear components of movement from a set of low-resolution interferograms, estimating at the same time the DEM error and the atmospheric artifacts. The basis for the linear estimation of movement is the adjustment of a linear model, which considers the linear velocity of displacement and the DEM error, to the available data in a similar way as done in the preceding methods [10], [12], [13]. The pixel selection criterion is based on its coherence stability in the stack of interferograms, in consequence the final product will have lower resolution than the original images and interferograms with short baselines will be preferred but this restriction is not compulsory. Besides this, the generation of the interferograms does not require establishing a master image, allowing free combinations of all available images. These two characteristics enable the algorithm to work with a small number of images, if compared with the requirements of the PS technique for instance [12]. Preliminary experiments provided good deformation maps from a reduced set of only seven images [16], these results were very similar to the ones obtained later on from a larger dataset of 20 images of the same zone [17]. This flexibility allows the user to generate deformation maps at a reduced cost and once a problematic zone is detected to plan the acquisition of more images. The method adjusts a linear model to phase increments between two neighboring pixels linked with the Delauney triangulation [18], avoiding the need of a sparse grid phase unwrapping of the interferograms.

Once the linear velocity of deformation and the DEM error have been retrieved, the algorithm continues with the nonlinear movement and the atmospheric artifacts estimation. In essence the algorithm takes advantage of the different behavior of the atmospheric artifacts in time and space with respect the nonlinear movement to isolate their respective contributions to the phase. Although the approach is similar in philosophy to all the methods previously noted, the practical implementation is different and oriented to strengthen the algorithm robustness. A combination of temporal and spatial filters sequentially applied are able to extract the atmospheric artifacts and the low- and highpass components of the nonlinear movement. As the interferograms were generated freely from the available images, the SVD method [10] is used to retrieve the temporal sequence suitable for the temporal filtering. One of the advantages of the algorithm is that there is no need to unwrap the noisy differential interferograms, which can be a difficult step and a potential source of errors. In addition the SVD method provides a minimum norm solution and allows the connection among nonconnected subsets of interferograms, however some fast nonlinear movements could be underestimate. The coupling of the atmosphere and the nonlinear movement, as both can present a similar phase behavior, is a common limitation in all methods.

In summary the processing main steps of the proposed algorithm are: selection of the image set covering the desired time interval, formation of the optimal inteferogram pairs depending on the maximum spatial baseline allowed, identification of the pixels candidates (those presenting a good phase quality) with a criterion based on its coherence stability along the interferograms stack, triangulation of the selected pixels to establish phase relations among them, and the adjustment of a linear model, which considers linear deformation and DEM error, to those phase relations for the whole set of interferograms. Once the linear movement has been estimated, the algorithm isolates the nonlinear component of movement from the atmospheric artifacts applying successive spatial and temporal filters. Finally, the results are integrated and interpolated to generate the movement maps.

The paper is organized as follows. Section II explains the extraction of the linear component of movement and the DEM error from the interferograms and Section III the nonlinear component and the atmospheric artifacts. Once the theoretical aspects have been discussed, Section IV introduces the European Remote Sensing (ERS) dataset used to validate the algorithm and Section $\mathrm{V}$ shows the results obtained in three different experiments. Finally, conclusions and open problems are addressed in Section VI.

\section{EXTRACTION OF LINEAR DEFORMATION}

When generating an interferogram by combining two SAR images, its phase variation between neighboring pixels can be expressed as [22]

$$
\delta \phi_{\mathrm{int}}=\delta \phi_{\mathrm{flat}}+\delta \phi_{\mathrm{topo}}+\delta \phi_{\mathrm{mov}}+\delta \phi_{\mathrm{atm}}+\delta \phi_{\mathrm{noise}}
$$

where $\delta \phi_{\text {flat }}$ earth is the flat earth component related with range distance; $\delta \phi_{\text {topo }}$ is the topographic phase; $\delta \phi_{\text {mov }}$ is the component due to the displacement of the terrain in range direction [line of sight (LOS)] between both SAR acquisitions; $\delta \phi_{\text {atm }}$ is the phase related with atmospheric artifacts; and $\delta \phi_{\text {noise }}$ comprises degradation factors related with temporal 
and spatial decorrelation and thermal noise. Three of the terms are analytically well known ( $\delta \phi_{\text {flat }}, \delta \phi_{\text {topo }}$, and $\left.\delta \phi_{\text {mov }}\right)$

$$
\begin{gathered}
\delta \phi_{\text {flat }}=\frac{4 \pi}{\lambda \cdot r} \cdot \frac{b \cdot \Delta r}{\tan \theta} \\
\delta \phi_{\text {topo }}=\frac{4 \pi}{\lambda \cdot r} \cdot \frac{b \cdot \Delta h}{\sin \theta} \\
\delta \phi_{\text {mov }}=\delta \phi_{\text {linear }}+\delta \phi_{\text {nonlinear }}=\frac{4 \pi}{\lambda} \cdot \Delta v \cdot T+\delta \phi_{\text {nonlinear }}
\end{gathered}
$$

where $\lambda$ is the wavelength; $r$ the range distance; $b$ the normal baseline (normal component of the separation between the position of satellites in both acquisitions respect to the incident angle); $\Delta r$ is the range increment between pixels; $\theta$ the incidence angle; $\Delta h$ and $\Delta v$ are the height and velocity increments between neighboring pixels respectively; and $T$ is the temporal baseline between both SAR acquisitions. Note that the deformation term has two contributions: linear and nonlinear displacement. By removing the flat earth and topographic terms we obtain the following DInSAR phase:

$$
\delta \phi_{\text {dif }}=\delta \phi_{\text {mov }}+\delta \phi_{\text {error topo }}+\delta \phi_{\text {atm }}+\delta \phi_{\text {noise }}
$$

As the DEM used to cancel the topography is not perfect, $\delta \phi_{\text {error topo }}$ is the phase component associated to the height error

$$
\delta \phi_{\text {error topo }}=\frac{4 \pi}{\lambda \cdot r} \cdot \frac{b \cdot \Delta \varepsilon}{\sin \theta}
$$

where $\Delta \varepsilon$ is the height error increment expressed in meters. If a set of differential interferograms of the same area is used, the topographic error and the linear deformation are correlated in all the images following (4) and (6). As the atmospheric conditions change from acquisition to acquisition, the atmospheric contribution behaves as a white process in time [21], [22]. Consequently, we can consider the atmospheric contribution and noise temporally decorrelated along the whole set of interferograms. With this approach, a model, which considers linear velocity deformation and DEM error, can be fitted to the set of differential interferograms with different temporal baselines. The linear deformation model cannot be applied to all the pixels within the area under study since only a part of them have sufficient phase quality due to decorrelation. Therefore, a first selection based on their phase quality estimation has to be performed before computing their deformation velocity. This selection can be done with two methods, one based on pixel amplitude stability and the other on spatial coherence. The selection based on amplitude presented in [12] and [13] estimates the phase standard deviation of every pixel from its temporal amplitude stability, which preserves the maximum spatial resolution of the images and allows detecting single isolated scatterers smaller than a resolution cell. One drawback of this technique is the large number of radiometrically calibrated images required, typically more than 30 [12], to successfully achieve a correct statistical study. On the other hand, the spatial coherence $\gamma$ is used to obtain the maximum likelihood estimator of the coherence magnitude [22] and provides an estimation of the accuracy of the pixel's phase for each interferogram not dependent on the number of images available. The required estimation window worsens the spatial resolution and can cause the loss of isolated scatterers which could be detected with the amplitude criteria. In our algorithm, pixels on

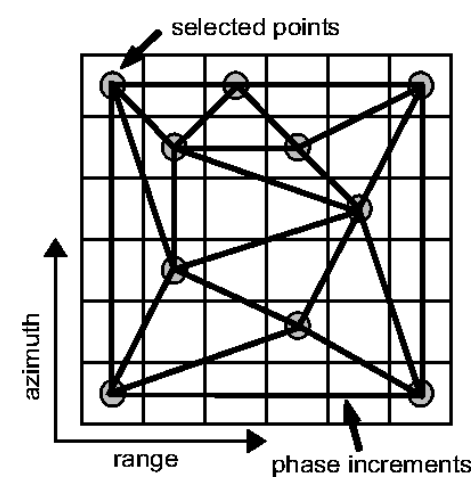

Fig. 1. Example of Delaunay triangulations.

multilooked images are selected from their coherence stability. A mean coherence image is generated from the whole stack of coherence maps in the following way:

$$
\gamma_{\text {mean }}=\frac{1}{N} \cdot \sum_{i=0}^{N-1} \gamma_{i}
$$

where $N$ is the number of interferograms. All pixels with a mean coherence over a selection threshold are accepted as candidates for the next step of the algorithm. A minimum value of mean coherence of 0.25 has been probed suitable for most of the cases. Note that this step is only a selection of candidates, and some of them will be rejected later if they do not adjust to the linear model. The size of the spatial window to calculate coherence sets the final resolution of the deformation maps. In our experiments, an averaging window of $4 \times 16$ or $5 \times 20$ (range $\times$ azimuth) has been probed enough for a good compromise between estimation of coherence and final resolution. If window dimensions are too small, the computed coherence will overestimate phase quality [22], and most of the pixels selected will be rejected on the next steps of the algorithm. We have to remember that the coherence is calculated once the fringes related with topography and flat earth have been eliminated; then, spatial coherence can be considered a good estimator.

The phase of individual pixels is not of practical utility due to the presence of different phase offsets among differential interferograms. These offsets could be calculated over high-coherence stable areas not affected by deformation and atmospheric artifacts, but in this case, additional input information would be required. This problem can be overcome relating two neighboring pixels, $\left(x_{m}, y_{m}\right)$ and $\left(x_{n}, y_{n}\right)$, by means of the Delaunay triangulation [18], [19] and canceling then the offsets effect. This kind of triangulations relates all the neighboring pixels of irregularly gridded data generating nonoverlapped triangles, see Fig. 1. Then, the differential phase increments can be expressed as

$$
\begin{aligned}
\delta \phi_{\text {dif }} & \left(x_{m}, y_{m}, x_{n}, y_{n}, T_{i}\right)=\frac{4 \pi}{\lambda} \cdot T_{i} \cdot\left[v\left(x_{m}, y_{m}\right)-v\left(x_{n}, y_{n}\right)\right] \\
& +\frac{4 \pi}{\lambda} \cdot \frac{b\left(T_{i}\right)}{r\left(T_{i}\right) \cdot \sin \left(\theta_{i}\right)} \cdot\left[\varepsilon\left(x_{m}, y_{m}\right)-\varepsilon\left(x_{n}, y_{n}\right)\right] \\
& +\left[\beta\left(x_{m}, y_{m}, T_{i}\right)-\beta\left(x_{n}, y_{n}, T_{i}\right)\right] \\
& +\left[\alpha\left(x_{m}, y_{m}, T_{i}\right)-\alpha\left(x_{n}, y_{n}, T_{i}\right)\right] \\
& +\left[n\left(x_{m}, y_{m}, T_{i}\right)-n\left(x_{n}, y_{n}, T_{i}\right)\right]
\end{aligned}
$$


where $x$ and $y$ are the pixel position coordinates within the image; $T_{i}$ is the time baseline of the $i$ th interferogram; $\lambda$ is the wavelength; $v$ is the constant velocity of the linear model of displacement; $b$ the spatial baseline of the $i$ th interferogram; $r$ the range distance; $\theta$ the incidence angle; $\varepsilon$ the topographic error; $\beta$ the nonlinear component of velocity; $\alpha$ the atmospheric phase artifacts; and $n$ decorrelation noise. Another advantage of relating neighboring pixels is that the atmospheric component is minimized for every relationship due to their spatial proximity. Taking into account that the atmospheric perturbation is a spatial small wavenumber signal, we can consider for neighboring pixels that

$$
\alpha\left(x_{m}, y_{m}, T_{i}\right) \approx \alpha\left(x_{n}, y_{n}, T_{i}\right) .
$$

The assumption considered in (9) holds if during triangulation the maximum distance allowed to connect two separate pixels is limited to approximately $1 \mathrm{~km}$, which is a reasonable correlation distance of the atmosphere [21], [22]. In some cases, nonconnected isolated areas will have to be studied independently. As the linear velocity and DEM error are constants in the whole set of differential interferograms, it is possible to retrieve a good estimation of them adjusting the following phase model to data [10], [12], [13]:

$$
\begin{aligned}
\delta \phi_{\text {model }} & \left(x_{m}, y_{m}, x_{n}, y_{n}, T_{i}\right) \\
= & \frac{4 \pi}{\lambda} \cdot T_{i} \cdot\left[v_{\text {model }}\left(x_{m}, y_{m}\right)-v_{\text {model }}\left(x_{n}, y_{n}\right)\right] \\
& +\frac{4 \pi}{\lambda} \cdot \frac{b\left(T_{i}\right)}{r\left(T_{i}\right) \cdot \sin \left(\theta_{i}\right)} \\
& \cdot\left[\varepsilon_{\text {model }}\left(x_{m}, y_{m}\right)-\varepsilon_{\text {model }}\left(x_{n}, y_{n}\right)\right] .
\end{aligned}
$$

This can be performed maximizing the following model coherence function [13]:

$$
\begin{aligned}
& \gamma_{\text {model }}\left(x_{m}, y_{m}, x_{n}, y_{n}\right) \\
&=\frac{1}{N} \cdot \mid \sum_{i=0}^{N} \exp \left[j \cdot \left(\delta \phi_{\text {dif }}\left(x_{m}, y_{m}, x_{n}, y_{n}, T_{i}\right)\right.\right. \\
&\left.\left.\quad-\delta \phi_{\text {model }}\left(x_{m}, y_{m}, x_{n}, y_{n}, T_{i}\right)\right)\right] \mid
\end{aligned}
$$

where $N$ is the number of interferograms. This function is equal to one when the adjustment to data is perfect and zero in case of total decorrelation. The maximization of the function is equivalent to find the bidimensional frequency of the complex sinusoid derived from the phase term (10). For each relation established by the Delauney triangulation, the values of this sinusoid are known over an irregular grid defined by the available pairs of temporal and spatial baselines, $\left\{b\left(T_{i}\right), T_{i}\right\}$. A reduced set of interferograms can cause an erroneous estimation of the unknown frequencies as different combinations of velocity and DEM error can generate similar phases. The larger the number of interferograms, the better will be the estimation as the range of multiple solutions is reduced. There is not a clear minimum number of images as results depends on each case, but we found that seven interferograms can provide good results and it is very difficult to work with less than five. Other interesting considerations about the maximization of (11) and its implicit constraints can be found in [13].
Once this maximization process has been done for each relationship, the result is the following set of velocity and topographic error increments:

$$
\begin{aligned}
& \Delta v_{\text {estimated }}\left(x_{m}, y_{m}, x_{n}, y_{n}\right) \\
& =\left[v_{\text {model }}\left(x_{m}, y_{m}\right)-v_{\text {model }}\left(x_{n}, y_{n}\right)\right]_{\text {maximize } \gamma} \\
& \begin{aligned}
\Delta \varepsilon_{\text {estimated }} & \left(x_{m}, y_{m}, x_{n}, y_{n}\right) \\
= & {\left[\varepsilon_{\text {model }}\left(x_{m}, y_{m}\right)-\varepsilon_{\text {model }}\left(x_{n}, y_{n}\right)\right]_{\text {maximize } \gamma} }
\end{aligned}
\end{aligned}
$$

At this point, a new quality test is performed and all the connections with a model coherence below a threshold are rejected. Good results have been obtained with coherence thresholds larger than 0.7 . As a consequence of the quality test, some pixels will be left isolated and also eliminated. An integration process is necessary to obtain absolute values for each pixel. We have used an approach based on the classical region growing algorithm for phase unwrapping [20]. The integration starts from different seed points, chosen from those presenting links with better model coherences, and calculates the absolute value of velocity for each pixel using

$$
\begin{aligned}
v_{\text {estimated }} & (x, y) \\
= & \frac{1}{\sum_{i} \gamma_{\text {model }}\left(x, y, x_{i}, y_{i}\right)} \\
& \cdot \sum_{i}\left[v_{\text {estimated }}\left(x_{i}, y_{i}\right)+\Delta v_{\text {estimated }}\left(x, y, x_{i}, y_{i}\right)\right] \\
& \cdot \gamma_{\text {model }}\left(x, y, x_{i}, y_{i}\right)
\end{aligned}
$$

where index $i$ corresponds to those neighboring pixels connected to the one that is being integrated. Each contribution reaching a pixel is weighted with its associated model coherence to reduce the contribution of the less reliable connections. A similar procedure is performed for the DEM error.

Fig. 2 shows a detailed layout of the method. In order to select the images covering a given time interval it is very important to have a homogeneous distribution of temporal baselines to correctly compute the velocity of deformation. Moreover, if the estimation of the DEM error is just an intermediate product and our goal is the correct measurement of deformation, the best results are obtained with a selection of short spatial baselines, which allow generate interferograms with lower spatial decorrelation and thus select a larger number of coherent pixels. Despite the DEM error estimation will be less reliable, as short baselines reduces their impact on phase, the estimation of movement will be more reliable. Fig. 3 shows the impact on the phase of different DEM errors as a function of the baseline length. Another important topic is the usage of multiple-pairs, common SAR images in different interferograms or in other words interferograms which are lineal combinations of others with respect the images used, from the available dataset. For instance, if we generate three interferograms from three images, each interferogram has different coherences and in practice one of them cannot be considered strictly as a lineal combination of the rest. This redundancy increases the number of data in the model coherence maximized (11) and allows a better estimation of the linear velocity 


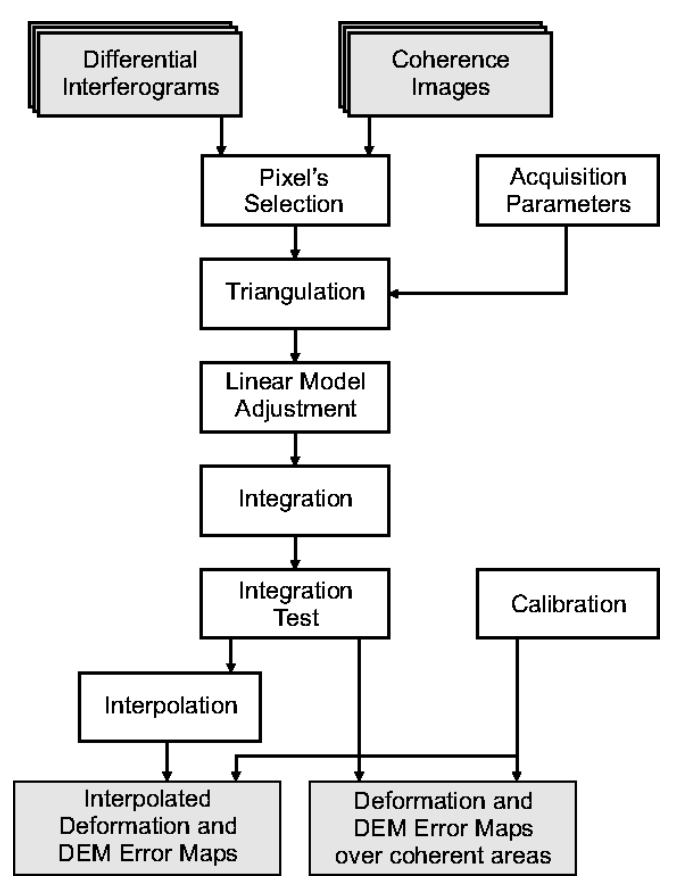

Fig. 2. Layout of the linear deformation algorithm.

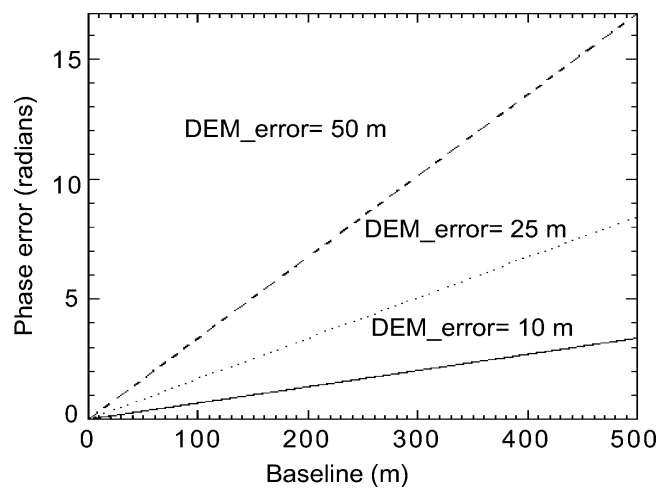

Fig. 3. Impact of the DEM errors on the phase error as a function of the baseline length.

and the DEM error even from a reduced set of images. It is also important to notice that orbit errors can be considered as a phase plane over a 100-km distance; therefore, under the point of view of the algorithm they will be treated as atmosphere [23].

\section{NONLINEAR DEFORMATION}

After calculating the linear deformation map, it is possible to obtain the nonlinear component to complete the study of displacement. Adding this nonlinear component to the linear term a detailed plot of the evolution of the deformation is obtained, as shown in Fig. 4. The first step is the calculation of the phase residues obtained by subtracting the previously estimated linear deformation and the DEM error from the original differential interferometric phases, as shown in the following equation:

$\delta \phi_{\text {residue }}\left(x, y, T_{i}\right)=\delta \phi_{\text {dif }}\left(x, y, T_{i}\right)-\delta \phi_{\text {linear model }}\left(x, y, T_{i}\right)$

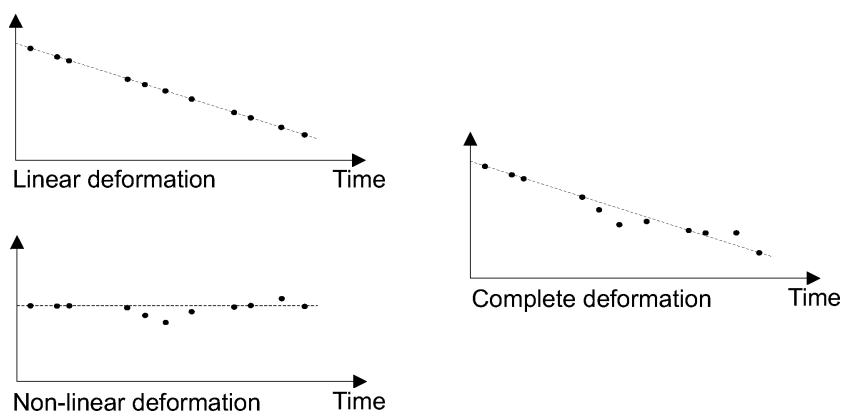

Fig. 4. Linear and nonlinear components of deformation.

where $\delta \phi_{\text {dif }}$ is the original differential phase, and $\delta \phi_{\text {linear model }}$ is the synthesized phase from the linear model, which considers the movement and the DEM error

$\delta \phi_{\text {linear model }}=\frac{4 \pi}{\lambda} \cdot T_{i} \cdot v_{\text {estimated }}+\frac{4 \pi}{\lambda} \cdot \frac{b}{r \cdot \sin \theta} \cdot \varepsilon_{\text {estimated }}$.

This procedure is carried out over the coherent pixels and it is followed by a spatial bilinear interpolation to get uniform pixel spacing. After this step, the phase residue can be expressed as

$$
\begin{aligned}
\delta \phi_{\text {residue }}\left(x, y, T_{i}\right)=\delta \phi_{\text {nonlinear }}\left(x, y, T_{i}\right) & \\
& +\delta \phi_{\text {atmos }}\left(x, y, T_{i}\right)+\delta \phi_{\text {decorr }}\left(x, y, T_{i}\right)
\end{aligned}
$$

where two important terms must be considered: the atmospheric artifacts $\delta \phi_{\text {atmos }}$ and the nonlinear displacement $\delta \phi_{\text {nonlinear }}$. Both terms can be isolated taking advantage of their different frequency characteristics in space and time. Atmospheric perturbations are considered as a low spatial frequency signal in each interferogram due to its approximately $1-\mathrm{km}$ correlation distance [21]; but for a given pixel its atmospheric contribution can be considered as a white process in time, because for each acquisition date atmospheric conditions can be considered a random variable. For instance, the characteristics of troposphere are different from one day to another. On the other hand, the nonlinear movement presents a narrower correlation window in space and a lowpass behavior in time. Taking into account all these considerations, the estimation of the atmospheric artifacts can be implemented with a filtering process in both spatial and time domains. Note that complete separation in frequency will not be possible due to the white process behavior of atmospheric artifacts.

The spatial lowpass filtering is carried out applying a two-dimensional moving averaging window of $1-\mathrm{km} \times 1-\mathrm{km}$ (typical correlation distance of atmosphere). After applying this filter to $\delta \phi_{\text {residue }}$, two components remain

$$
\begin{aligned}
\delta \phi_{\text {residue SLR }}\left(x, y, T_{i}\right)=\delta & \phi_{\text {atmos }}\left(x, y, T_{i}\right)+ \\
& +\delta \phi_{\text {nonlinear SLR }}\left(x, y, T_{i}\right)
\end{aligned}
$$

where $\delta \phi_{\text {nonlinear SLR }}$ is the nonlinear component of the displacement at spatial low resolution (SLR), and it is assumed that atmosphere $\delta \phi_{\text {atmos }}$ is not affected by the spatial filter. After this step, a temporal highpass filter should be applied to retrieve an important part of atmospheric contribution. The interferograms have been formed from the phases of two SAR images separated in time, and, as noted before, no temporal restriction has been applied on the pair selection. Consequently, the interferograms 
obtained do not follow the temporal order required by the filter. The formation of the interferograms can be expressed in the following way:

$$
\begin{aligned}
& \delta \phi_{\text {residue } \mathrm{SLR}}\left(x, y, T_{i}\right)=\phi_{\text {residue } \mathrm{SLR}}\left(x, y, t_{j}\right) \\
& -\phi_{\text {residue } \operatorname{SLR}}\left(x, y, t_{k}\right) \quad \forall i=1, \ldots, N
\end{aligned}
$$

where $N$ is the number of interferograms and $T_{i}=t_{k}-t_{j}$, being $t_{k}$ and $t_{j}$ the acquisition times of both SAR images (master and slave). Equation (19) if expressed as a system of equations can be solved to retrieve the phase contribution of each single image. In the most general case the SLR residual phases have to be unwrapped prior to solving the system. This heavily filtered interferograms are especially easy to unwrap with the classical methods, as they are very smooth and contain almost no fringes. The SLR residue is composed by the atmospheric perturbations, which are signals with a low variation in space, and the spatial low-resolution version of the nonlinear displacement, which behaves in space similarly to the atmospheric component. In this case, the least mean squares (LMS) method has been used [24]. Once the SLR residues have been unwrapped, it is possible to solve the system of equations (19). The matrix to invert is singular when disconnected subsets of interferograms, having no common SAR images, are present and then the system has an infinite number of solutions. For this reason, the singular value decomposition (SVD) method has been used as obtains the least square solution of minimum norm [9]-[11]. Then, the result is an approximation of the reality. Nevertheless, if the nonlinear displacement change between temporally adjacent samples is considered smooth, the correct phases will be retrieved. The algorithm will underestimate the nonlinear movement if very abrupt wide area displacements occur between disconnected subsets [10]. Once the system has been solved we get the phases belonging to each SAR image respect to the master

$$
\begin{aligned}
& \phi_{\text {residue } \operatorname{SLR}}\left(x, y, t_{i}\right) \\
= & \begin{cases}0, & i=0 \\
\phi_{\text {atmos }}\left(t_{i}\right)+\phi_{\text {nonlinear } \operatorname{SLR}}\left(t_{i}\right), & 1 \leq i \leq M-1\end{cases}
\end{aligned}
$$

where $M$ is the number of SAR images. Finally, a Kaiser temporal filter [25] is applied to reduce the atmospheric contribution and isolate the nonlinear deformation at low resolution. A key parameter is the selection of the cut frequency to discriminate the atmospheric component from the nonlinear displacement, as the atmospheric effects appear in all the frequencies while the nonlinear displacement is a lowpass signal. Therefore, the cut frequency should be placed on the considered highest frequency for the nonlinear displacement. With no a priori information it is difficult to estimate this frequency; in our case with moderate nonlinear displacements a $25 \%$ of the band has produced good results

$$
\begin{aligned}
& \phi_{\text {residue } \operatorname{SLR}}\left(x, y, t_{i}\right)_{\mathrm{LPF}} \\
= & \begin{cases}0, & i=0 \\
\phi_{\text {nonlinearSLR }}\left(t_{i}\right), & 1 \leq i \leq M-1 .\end{cases}
\end{aligned}
$$

Note that this displacement phase corresponds to each SAR acquisition, and added to the linear one gives us the complete evolution of the deformation. In a similar way, an approximation of the atmospheric contribution on each SAR image is obtained with a highpass temporal filter.

This information is not complete, because the SLR nonlinear deformation has been calculated using spatial lowpass-filtered phases. Some details of movement at spatial high resolution (SHR) have been omitted. The procedure to obtain the SHR component is similar to the one used to estimate the atmospheric artifacts and the SLR displacement. First, a better phase model using all the available information, including the estimated linear and SLR nonlinear deformation, the DEM error and the atmospheric contribution is generated

$$
\begin{aligned}
& \delta \phi_{\text {model }}\left(x, y, T_{i}\right) \\
&= \frac{4 \pi}{\lambda} \cdot T_{i} \cdot v_{\text {estimated }}+\delta \phi_{\text {nonlinear SLR }}\left(T_{i}\right) \\
&+\frac{4 \pi}{\lambda} \cdot \frac{b\left(T_{i}\right)}{r\left(T_{i}\right) \cdot \sin \left(\theta_{i}\right)} \cdot \varepsilon_{\text {estimated }}+\delta \phi_{\text {atmos }}\left(T_{i}\right) \\
& \quad \forall i=1, \ldots, N \quad
\end{aligned}
$$

where $\delta \phi_{\text {nonlinear SLR }}$ are the interferometric phases of the SLR nonlinear displacement generated in the following way:

$$
\begin{array}{r}
\delta \phi_{\text {nonlinear } \operatorname{SLR}}\left(x, y, T_{i}\right) \\
=\phi_{\text {nonlinear } \operatorname{SLR}}\left(x, y, t_{j}\right)-\phi_{\text {nonlinear } \operatorname{SLR}}\left(x, y, t_{k}\right) \\
\forall i=1, \ldots, N .
\end{array}
$$

A new residue can be obtained by subtracting the model from the original interferometric phases. The residue is basically composed by two terms

$$
\begin{aligned}
& \delta \phi_{\text {residue } \operatorname{SHR}}\left(x, y, T_{i}\right) \\
& \quad=\delta \phi_{\text {nonlinear SHR }}\left(x, y, T_{i}\right)+\delta \phi_{\text {decorr }}\left(x, y, T_{i}\right)
\end{aligned}
$$

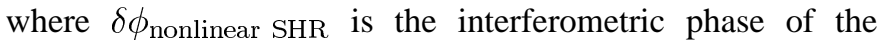
SHR nonlinear displacement, and $\delta \phi_{\text {decorr }}$ is a term related with decorrelation and thermal noise. If we assume that the decorrelation term is much smaller than the SHR nonlinear displacement, we can solve a system of equations formed from (25) to obtain the movement information for each single image

$$
\begin{aligned}
& \delta \phi_{\text {residue SHR }}\left(x, y, T_{i}\right)= \phi_{\text {residue } \operatorname{SHR}}\left(x, y, t_{j}\right) \\
&-\phi_{\text {residue } \operatorname{SHR}}\left(x, y, t_{k}\right) \\
& \forall i=1, \ldots, N .
\end{aligned}
$$

As it was done before, a phase unwrapping of the residues is compulsory before solving the system of equations with the SVD method. Nevertheless, we can consider this residue, associated with the nonlinear component of displacement, small enough to assume that is not larger than one phase cycle. This is an important assumption because phase unwrapping of these phases would require specific algorithms for sparse data [19]. Our experience using two different datasets and a simulator has shown this assumption is usually true. Then, the system can be solved with no need of phase unwrapping. The solution provides the phases associated to the nonlinear movement with the first image as the phase reference

$$
\begin{aligned}
\phi_{\text {residue SHR }}\left(x, y, t_{i}\right) \\
= \begin{cases}0, & i=0, \\
\phi_{\text {nonlinear } \operatorname{SHR}}\left(x, y, t_{i}\right), & 1 \leq i \leq M-1 .\end{cases}
\end{aligned}
$$




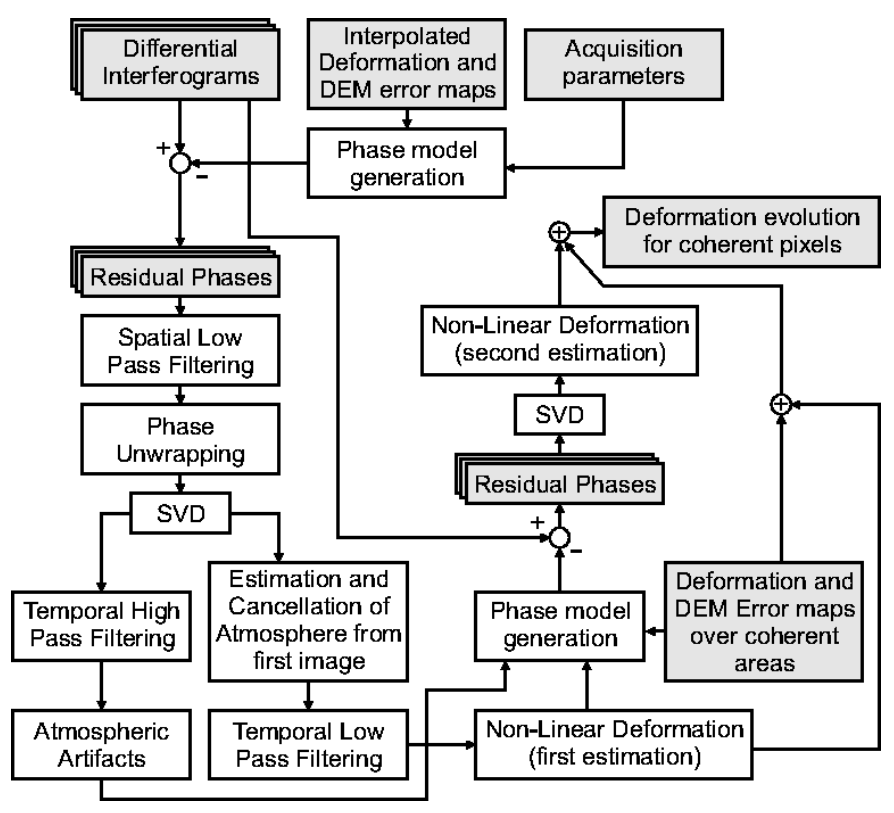

Fig. 5. Layout of the nonlinear deformation algorithm.

The same considerations pointed out when SVD was applied before are still valid. Finally, the complete evolution of deformation is obtained considering all the components previously estimated

$$
\begin{aligned}
\rho\left(t_{i}\right)=\frac{\lambda}{4 \pi} \cdot & {\left[\frac{4 \pi}{\lambda} \cdot\left(t_{i}-t_{o}\right) \cdot v_{\text {estimated }}\right.} \\
& \left.+\phi_{\text {nonlinearSLR }}\left(t_{i}\right)+\phi_{\text {nonlinearSHR }}\left(t_{i}\right)\right] .
\end{aligned}
$$

A detailed layout of the procedure for the nonlinear estimation of displacement is shown in Fig. 5.

\section{DATA}

The algorithm is able to provide good results even with a reduced set of SAR images, for instance preliminary results were obtained with a dataset of only seven SAR images [16]. In this paper, a set of 23 ERS SAR images, ranging from 1992 to 1999, has been used, as shown in Table I. The images were chosen to form 24 interferograms with short spatial baselines, between 2 and $24 \mathrm{~m}$ (see Table II). A patch of 10-km $\times 16-\mathrm{km}$ from the whole frame, which contains two interesting towns, has been selected. The small town on the upper left corner of the SAR image shown in Fig. 6 has subsidence problems, while the bigger bright zone on the lower corner can be considered a stable area.

The images have been multilooked, with a pixel resolution in ground range of $100 \times 100 \mathrm{~m}$. A commercial DEM of the zone, provided by the Institut Cartogràfic de Catalunya (ICC), has been used to remove the topographic component. Fig. 6 shows the interferometric phase and coherence obtained from the pair formed with the images acquired July 23, 1997, and August 12, 1998. The large coherent areas correspond to urban zones, where coherence is better preserved, while the rest of the image mostly corresponds to vegetated areas with lower coherence due to temporal decorrelation.
TABLE I

LIST OF THE SAR IMAGES USED IN THIS STUDY

\begin{tabular}{ccrcc}
\hline & Mission & Orbit & $\begin{array}{c}\text { Date } \\
\text { (day/month/year) }\end{array}$ & $B_{\perp}$ (meters) \\
\hline 1 & ERS-1 & 7081 & $22 / 11 / 1992$ & 0 \\
2 & ERS-1 & 8083 & $31 / 01 / 1993$ & -818 \\
3 & ERS-1 & 12091 & $07 / 11 / 1993$ & 135 \\
4 & ERS-1 & 20451 & $13 / 06 / 1995$ & -416 \\
5 & ERS-1 & 20952 & $18 / 07 / 1995$ & -263 \\
6 & ERS-1 & 21954 & $26 / 09 / 1995$ & -242 \\
7 & ERS-2 & 2281 & $27 / 09 / 1995$ & 112 \\
8 & ERS-2 & 2782 & $01 / 11 / 1995$ & 460 \\
9 & ERS-1 & 24960 & $23 / 04 / 1996$ & 464 \\
10 & ERS-1 & 25461 & $28 / 05 / 1996$ & -524 \\
11 & ERS-2 & 6289 & $03 / 07 / 1996$ & -9 \\
12 & ERS-2 & 7291 & $11 / 09 / 1996$ & -529 \\
13 & ERS-2 & 9295 & $29 / 01 / 1997$ & 23 \\
14 & ERS-2 & 11800 & $23 / 07 / 1997$ & -233 \\
15 & ERS-2 & 12301 & $27 / 08 / 1997$ & 140 \\
16 & ERS-2 & 14806 & $18 / 02 / 1998$ & -422 \\
17 & ERS-2 & 15808 & $29 / 04 / 1998$ & 31 \\
18 & ERS-2 & 16810 & $08 / 07 / 1998$ & -807 \\
19 & ERS-2 & 17311 & $12 / 08 / 1998$ & -239 \\
20 & ERS-2 & 17812 & $16 / 09 / 1998$ & -831 \\
21 & ERS-2 & 19816 & $03 / 02 / 1999$ & -515 \\
22 & ERS-2 & 21319 & $19 / 05 / 1999$ & -480 \\
23 & ERS-1 & 41994 & $27 / 07 / 1999$ & 454 \\
\hline
\end{tabular}

TABLE II

LIST OF THE SET OF 24 SHORT BASELINE INTERFEROGRAMS

\begin{tabular}{cccc}
\hline & $\begin{array}{c}\text { Master Date } \\
\text { (day/month/year) }\end{array}$ & $\begin{array}{c}\text { Slave Date } \\
\text { (day/month/year) }\end{array}$ & $B_{\perp}$ (meters) \\
\hline 1 & $22 / 11 / 1992$ & $03 / 07 / 1996$ & 8 \\
2 & $22 / 11 / 1992$ & $29 / 01 / 1997$ & -24 \\
3 & $31 / 01 / 1993$ & $08 / 07 / 1998$ & -11 \\
4 & $31 / 01 / 1993$ & $16 / 09 / 1998$ & 13 \\
5 & $07 / 11 / 1993$ & $27 / 09 / 1995$ & 23 \\
6 & $07 / 11 / 1993$ & $27 / 08 / 1997$ & -6 \\
7 & $13 / 06 / 1995$ & $18 / 02 / 1998$ & 7 \\
8 & $18 / 07 / 1995$ & $26 / 09 / 1995$ & -21 \\
9 & $18 / 07 / 1995$ & $12 / 08 / 1998$ & -24 \\
10 & $18 / 07 / 1995$ & $19 / 05 / 1999$ & -19 \\
11 & $26 / 09 / 1995$ & $23 / 07 / 1997$ & -9 \\
12 & $26 / 09 / 1995$ & $12 / 08 / 1998$ & -3 \\
13 & $26 / 09 / 1995$ & $19 / 05 / 1999$ & 2 \\
14 & $01 / 11 / 1995$ & $23 / 04 / 1996$ & -4 \\
15 & $01 / 11 / 1995$ & $27 / 07 / 1999$ & 5 \\
16 & $23 / 04 / 1996$ & $27 / 07 / 1999$ & 9 \\
17 & $28 / 05 / 1996$ & $11 / 09 / 1996$ & 5 \\
18 & $28 / 05 / 1996$ & $03 / 02 / 1999$ & -10 \\
19 & $11 / 09 / 1996$ & $03 / 02 / 1999$ & -15 \\
20 & $29 / 01 / 1997$ & $29 / 04 / 1998$ & -8 \\
21 & $23 / 07 / 1997$ & $12 / 08 / 1998$ & 6 \\
22 & $23 / 07 / 1997$ & $19 / 05 / 1999$ & 11 \\
23 & $08 / 07 / 1998$ & $16 / 09 / 1998$ & 24 \\
24 & $12 / 08 / 1998$ & $19 / 05 / 1999$ & 5 \\
\hline
\end{tabular}

\section{RESULtS}

The first step of the processing is the selection of those pixels that present coherence stability in time. In this case, pixels having a mean coherence value higher than 0.25 have been selected. The result is a set of 1437 pixels distributed all over the image. Obviously, the density of points is higher in the urban areas than in the vegetated ones. All these pixels are related using the Delaunay triangulation (Fig. 7). 

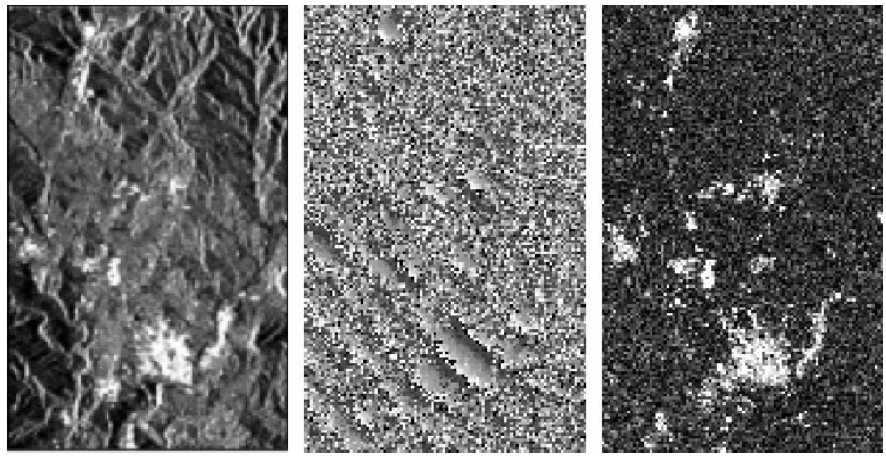

Fig. 6. (Left) SAR amplitude image, (center) interferometric phase, and (right) coherence map of the zone under study.

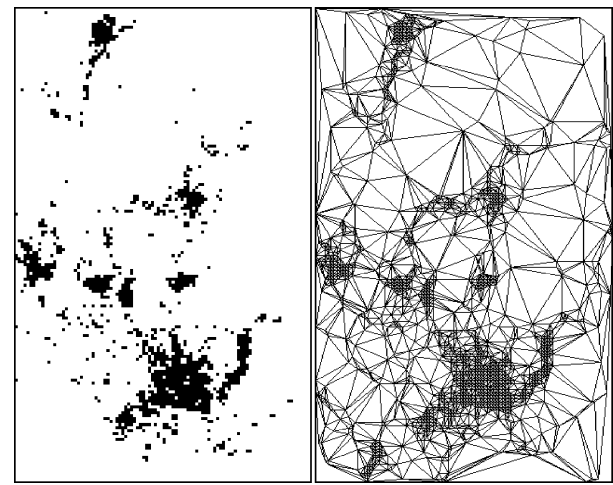

Fig. 7. Selected points using (left) the coherence criterion and (right) the Delaunay Triangulations before removing the links over 1-km length.

After processing the data using the algorithm for linear deformation estimation, the remaining number of points after the quality test, which checks its linearity, is 1236 from the initial 1437 selected. In addition to noisy pixels, some very nonlinearly moving quality points could have been eliminated and consequently lost. An important assumption of the method is that only nonlinear points free of strong abrupt deformations will pass all the quality filters. As it can be observed on the LOS (or slant-range) deformation velocity map in Fig. 8, only two small areas located in the upper left corner of the image present a subsidence velocity larger than $1 \mathrm{~cm}$ per year. The lower area, labeled as A, belongs to a suburb were subsidence has been causing structural damages in several buildings. The result over the bigger town in the lower part of the image (D), which corresponds to a stable area, shows the good behavior of the algorithm. Detailed deformation velocity maps over these two areas are shown in Fig. 9. The results over the suburb (A) have been validated with precise leveling measurements provided by ICC, who has been monitoring the zone. These measurements showed a maximum of subsidence of about $2 \mathrm{~cm}$ per year projected in the slant range direction in the same geographical position where the maximum of $1.8 \mathrm{~cm}$ per year (LOS) has been detected. Note that the horizontal deformation component cannot be calculated using only one orbital direction. For example, this term could be estimated using ascending and descending orbits [14].

Once the linear deformation has been obtained, the nonlinear deformation estimation algorithm has been applied to retrieve the full deformation evolution. Fig. 10 shows the four points (A,

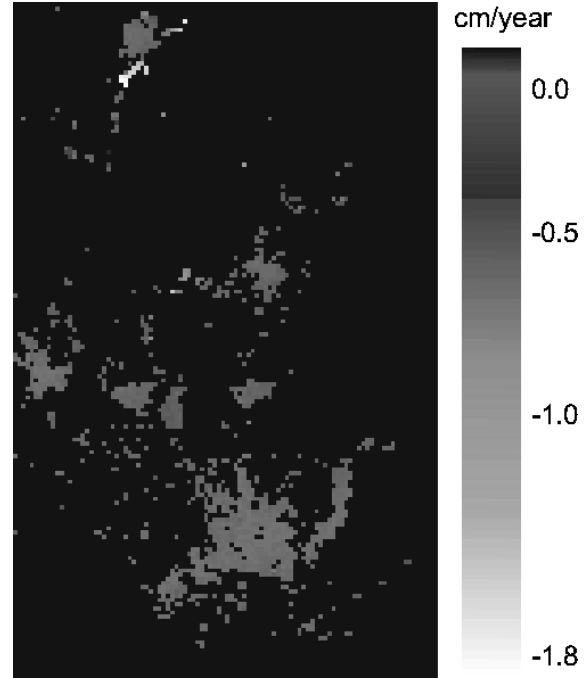

Fig. 8. Slant range deformation velocity map ( $\mathrm{cm}$ per year) of the area under study.
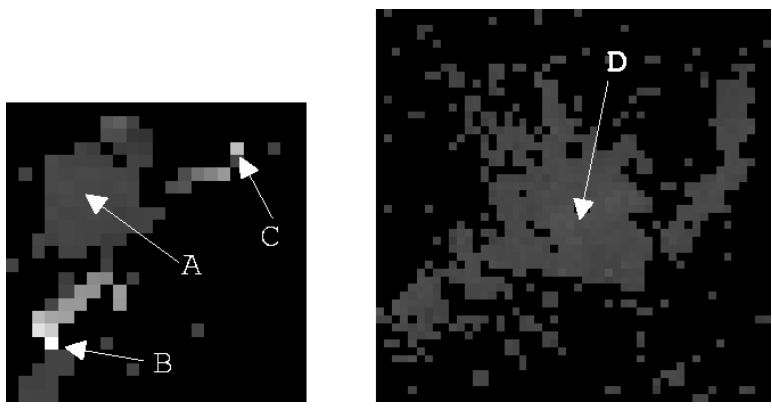

Fig. 9. Detailed deformation maps of the two towns, the left one affected by localized subsidence and the right one stable. The maximum deformation (white) corresponds to a velocity of $1.8 \mathrm{~cm}$ per year.

B, C, and D) depicted in Fig. 8. Point A, with the largest deformation, presents a very linear trend of displacement from 1992 to 1998 , but after 1998 the deformation seems to be smaller. On the other hand, point $\mathrm{B}$ shows a linear trend during the whole period of time. Points $C$ and $D$, located in stable urban areas, do not present remarkable movements, as it was expected.

To better evaluate the flexibility of the algorithm with respect the dataset requirements in both number of images and spatial baseline length, two additional tests have been carried out. The first one consisted in the reduction of the number of SAR images available, while keeping the short spatial baseline restriction on the selection of the interferometric pairs. In this case, 10 differential interferograms were generated using only 14 of the SAR images available (see Table III). The second test has also reduced the number of SAR images to 14 , but has increased the spatial baseline range in the 16 interferograms from 6 up to 402 $\mathrm{m}$ (see Table IV). For both cases, the obtained deformation velocity maps are shown in Fig. 11. The reduction of the dataset but keeping the low spatial baseline restriction on interferogram generation causes a slight reduction on the final number of points where deformation have been calculated, 1015 with 10 interferograms compared with the 1236 with 24 interferograms. Despite this, reduction is not noticeable over large urban areas where coherence is high; it can cause the loss of isolated pixels embedded in low-coherence areas. As expected, the larger the 

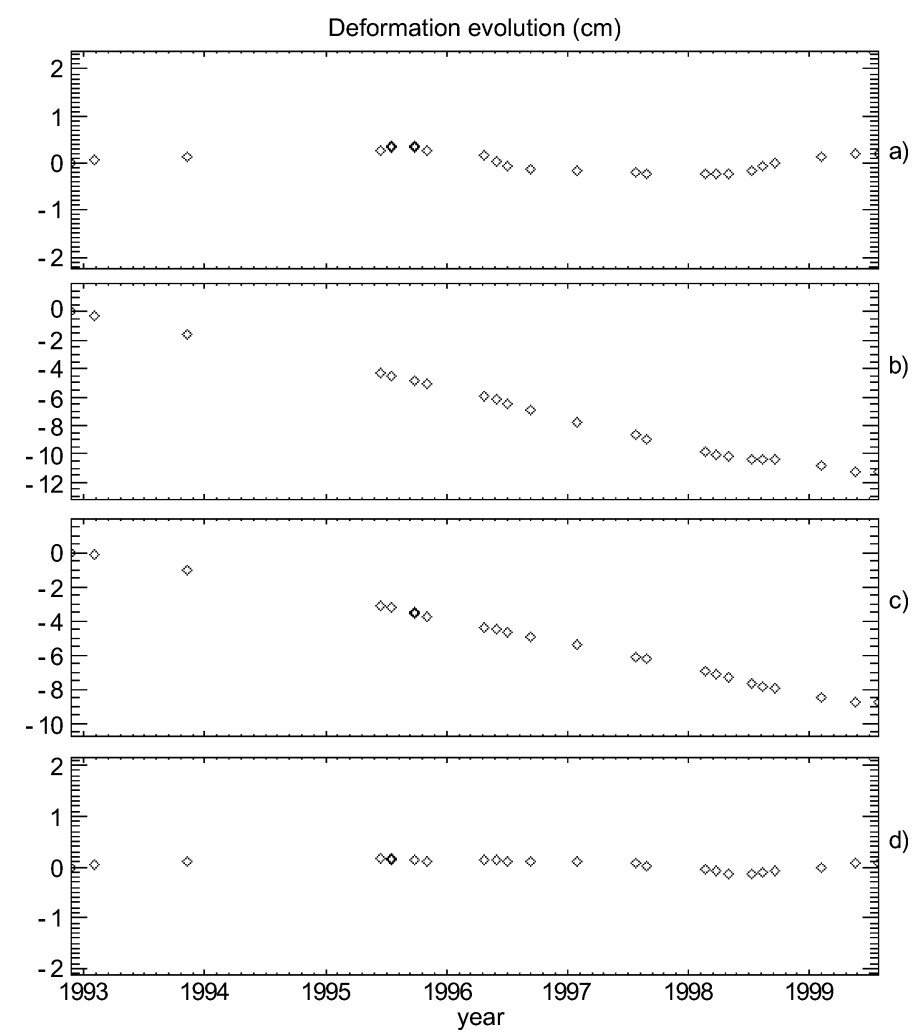

Fig. 10. Deformation evolution of points detailed in Fig. 9.

TABLE III

LIST OF THE REDUCED SET OF TEN SHORT BASELINE INTERFEROGRAMS

\begin{tabular}{cccc}
\hline & $\begin{array}{c}\text { Master Date } \\
\text { (day/month/year) }\end{array}$ & $\begin{array}{c}\text { Slave Date } \\
\text { (day/month/year) }\end{array}$ & $B_{\perp}$ (meters) \\
\hline 1 & $22 / 11 / 1992$ & $03 / 07 / 1996$ & 8 \\
2 & $22 / 11 / 1992$ & $29 / 01 / 1997$ & -24 \\
3 & $31 / 01 / 1993$ & $08 / 07 / 1998$ & -11 \\
4 & $31 / 01 / 1993$ & $16 / 09 / 1998$ & 13 \\
5 & $07 / 11 / 1993$ & $27 / 09 / 1995$ & 23 \\
6 & $07 / 11 / 1993$ & $27 / 08 / 1997$ & -6 \\
7 & $13 / 06 / 1995$ & $18 / 02 / 1998$ & 7 \\
8 & $18 / 07 / 1995$ & $26 / 09 / 1995$ & -21 \\
9 & $18 / 07 / 1995$ & $12 / 08 / 1998$ & -24 \\
10 & $18 / 07 / 1995$ & $19 / 05 / 1999$ & -19 \\
\hline
\end{tabular}

number of SAR short baseline interferograms the better the results. Fig. 12 shows a comparison between deformation evolution plots using 24 and 10 short baseline differential interferograms. Note the great agreement between both results. On the other hand, the effect of increasing the spatial baselines becomes more important. For the large baseline test, the number of points is reduced drastically, falling down to 478 from the initial 1236 even over cities. Taking into account that we maintain low spatial resolution (multilooked data) a high number of points are lost due to spatial decorrelation. As a consequence, the deformation areas are not detected in this case. When increasing spatial baseline to the limit of critical value, only point-like scatterers remain with enough quality. In this case, the selection criteria based on coherence cannot be used.
TABLE IV

LIST OF THE SET OF 16 COMBINED LARGE AND SHORT BASELINE INTERFEROGRAMS

\begin{tabular}{cccc}
\hline & $\begin{array}{c}\text { Master Date } \\
\text { (day/month/year) }\end{array}$ & $\begin{array}{c}\text { Slave Date } \\
\text { (day/month/year) }\end{array}$ & $B_{\perp}$ (meters) \\
\hline 1 & $22 / 11 / 1992$ & $03 / 07 / 1996$ & 8 \\
2 & $22 / 11 / 1992$ & $29 / 01 / 1997$ & -24 \\
3 & $31 / 01 / 1993$ & $08 / 07 / 1998$ & -11 \\
4 & $31 / 01 / 1993$ & $16 / 09 / 1998$ & 13 \\
5 & $07 / 11 / 1993$ & $27 / 09 / 1995$ & 23 \\
6 & $07 / 11 / 1993$ & $27 / 08 / 1997$ & -6 \\
7 & $13 / 06 / 1995$ & $18 / 02 / 1998$ & 7 \\
8 & $18 / 07 / 1995$ & $26 / 09 / 1995$ & -21 \\
9 & $18 / 07 / 1995$ & $12 / 08 / 1998$ & -24 \\
10 & $18 / 07 / 1995$ & $19 / 05 / 1999$ & -19 \\
11 & $27 / 09 / 1995$ & $01 / 11 / 1995$ & 348 \\
12 & $22 / 11 / 1992$ & $27 / 09 / 1995$ & 112 \\
13 & $23 / 07 / 1997$ & $29 / 04 / 1998$ & 264 \\
14 & $13 / 06 / 1995$ & $23 / 07 / 1997$ & 183 \\
15 & $31 / 01 / 1993$ & $13 / 06 / 1995$ & 402 \\
16 & $16 / 09 / 1998$ & $03 / 02 / 1999$ & 316 \\
\hline
\end{tabular}
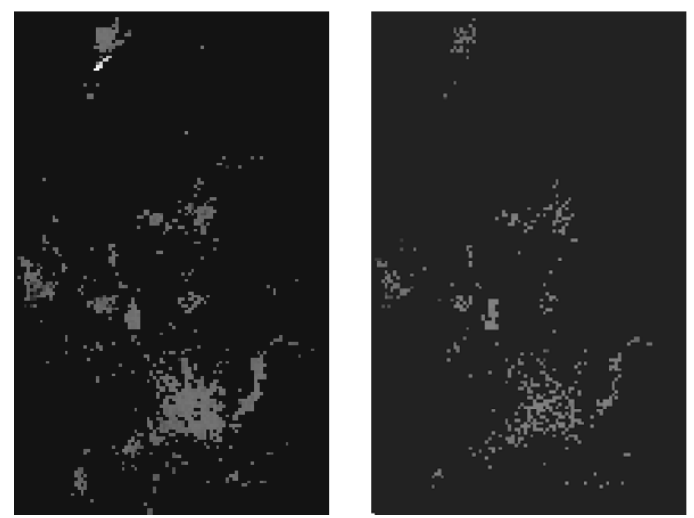

0.3

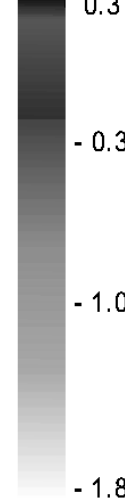

Fig. 11. Deformation maps using ten short baseline, up to $25 \mathrm{~m}$, (left) interferograms and (right) 16 large baseline, up to $402 \mathrm{~m}$, interferograms.

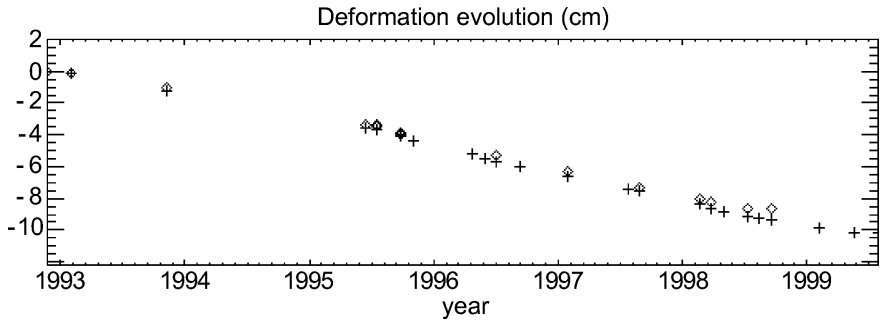

Fig. 12. Comparison of deformation plots obtained with 24 short baseline interferograms (crosses) and ten short baseline interferograms (diamonds).

\section{CONCLUSION}

A method with flexible requirements for the estimation of linear and nonlinear surface displacements from a set of differential interferograms has been presented. The number of SAR images required for a good performance of the algorithm can be considered low, if compared with other methods of the literature, basically due to the pixel selection criterion, based on a coherence threshold, and the nonrestricted generation of the interferometric pairs not constrained to follow any specific rule like all the combinations done with the same master image. These properties make the algorithm very convenient for detecting terrain 
movements from small datasets of images, allowing identifying the problematic zones at a reduced cost. Even though a reduced set of only seven images can provide a good estimation of the linear component of movement, the redundancy provided by the usage of larger datasets extends the penetration of the algorithm further into more difficult areas largely affected by temporal decorrelation. The benefits of increasing the number of images are more noticeable when retrieving the nonlinear component of movement, as this feature is closely related with the temporal sampling imposed by the image selection.

The pixel candidates selection criterion based on coherence limits the final resolution of the obtained deformation maps to the size of the used window, around $100 \times 100 \mathrm{~m}$ in ground in this paper. In addition, this criterion also introduces a practical limit on the baselines length to be used when generating the interferograms. The larger are the baselines, the higher is the spatial decorrelation, the more critical is the topography cancellation with a DEM and the lower the computed coherence. Then, all pixels containing point like structures presenting higher phase quality along time will be lost if surrounded by low-quality ones. The selection criterion allows reduce the number of required images, but worsens the final resolution and is not able to detect some good candidates surrounded by decorrelated areas. The algorithm performs better with short baseline interferograms. The initial set of candidates is later evaluated depending on its adjustment to the linear model of movement; as a consequence those few pixels presenting localized higher nonlinear movements will be regarded as noise, rejected by the algorithm and consequently lost.

The algorithm has been validated with data acquired by ESA's ERS-1/2 satellites from 1992 to 1999 . The area under study is a difficult test site due to the presence of large vegetated areas surrounding small populated zones. Nevertheless, the final results perfectly show the small areas of deformation controlled by the Institut Cartogràfic de Catalunya. The rest is stable, as it was expected. Finally, the complete deformation plot for all the coherent points has been calculated, showing different displacement trends due to nonlinear movement in the two small zones affected by subsidence.

Several issues are still open for further improvement. One of them is to find new selection criteria working with a reduced number of images which ensures pixel quality independently of its later linear behavior. These new criteria should allow to preserve the original image resolution and deal with highly nonlinear movements. Another problem that should be addressed in the future is the isolation of the coupled nonlinear deformation and atmospheric components. A simple temporal filtering is not enough to totally separate both components and an accurate evaluation of the coupling effects and the usage of a priori information should be considered.

\section{ACKNOWLEDGMENT}

The authors would like to thank the Institut Cartogràfic de Catalunya for providing a DEM of the studied area and the precise leveling measurements of terrain deformation used for validating the algorithm. They also thank the anonymous reviewers for their comments and suggestions.

\section{REFERENCES}

[1] D. Massonet et al., "The displacement field of the Landers earthquake mapped by radar interferometry," Nature, vol. 364, pp. 138-142, July 1993.

[2] G. Peltzer and P. A. Rosen, "Surface displacement of the 17 Eureka valley, California, earthquake observed by SAR interferometry," Science, vol. 268, pp. 1333-1336, June 1995.

[3] D. Massonet, P. Briole, and A. Arnaud, "Deflation of Mount Etna monitored by spacebrone radar interferometry," Nature, vol. 375, pp. 567-570, June 1995.

[4] T. Nagler, H. Rott, and A. Kamelger, "Analysis of landslides in Alpine areas by means of SAR interferometry," in Proc. IGARSS, vol. 1, Toronto, ON, Canada, July 2002, pp. 198-200.

[5] D. T. Sandwell and E. J. Price, "Phase gradient approach to stacking interferograms," J. Geophys. Res., vol. 103, pp. 30 183-30 204, 1998.

[6] S. Lyons and D. Sandwell, "Fault creep along the southern San Andreas from InSAR, permanent scatterers and stacking," J. Geophys. Res., vol. 108, no. 1, 2003.

[7] P. Lundgren et al., "Modeling surface deformation observed with SAR interferometry at Campi Flegrei caldera," J. Geophys. Res., vol. 106, pp. 19355-19367, Sept. 2001

[8] S. Usai, "A least-squares approach for long-term monitoring of deformations with differential SAR interferometry," in Proc. IGARSS 2002, vol. 2, Toronto, ON, Canada, June 2002, pp. 1247-1250.

[9] P. Berardino, G. Fornaro, A. Fusco, D. Galluzzo, R. Lanari, E. Sansosti, and S. Usai, "A new approach for analyzing the temporal evolution of Earth surface deformations based on the combination of DIFSAR interferograms," in Proc. IGARSS, vol. 6, Sydney, Australia, July 2001, pp. 2551-2553.

[10] P. Berardino, G. Fornaro, R. Lanari, and E. Sansosti, "A new algorithm for surface deformation monitoring based on small baseline differential interferograms," IEEE Trans. Geosci. Remote Sensing, vol. 40, pp. 2375-2383, Nov. 2002

[11] O. Mora, R. Lanari, J. J. Mallorquí, P. Berardino, and E. Sansosti, "A new algorithm for monitoring localized deformation phenomena based on small baseline differential SAR interferograms," in Proc. IGARSS, Toronto, ON, Canada, June 2002, pp. 1237-1239.

[12] A. Ferretti, C. Prati, and F. Rocca, "Permanent scatterers in SAR interferometry," IEEE Trans. Geosci. Remote Sensing, vol. 39, pp. 8-30, Jan. 2001.

[13] — , "Nonlinear subsidence rate estimation using permanent scatterers in differential SAR interferometry," IEEE Trans. Geosci. Remote Sensing, vol. 38, pp. 2202-2212, Sept. 2000.

[14] C. Colesanti, A. Ferretti, C. Prati, and F. Roca, "Full exploitation of the ERS archive: Multi data set permanent scatterers analysis," in Proc. IGARSS, vol. 2, Toronto, ON, Canada, July 2002, pp. 124-1236.

[15] A. Refice, F. Bovenga, L. Guerreiro, and J. Wasowski, "DInSAR applications to landslide studies," in Proc. IGARSS, vol. 1, Sydney, Australia, July 2001 , pp. 144-146.

[16] O. Mora, J. J. Mallorqui, J. Duro, and A. Broquetas, "Long-term subsidence monitoring of urban areas using differential interferometric SAR techniques," in Proc. IGARSS, vol. 3, Sydney, Australia, July 2001, pp. 1104-1106.

[17] O. Mora, J. J. Mallorqui, and J. Duro, "Generation of deformation maps at low resolution using differential interferometric SARdata," in Proc. IGARSS, vol. 5, Toronto, ON, Canada, July 2002, pp. 2696-2698.

[18] B. Delaunay, "Sur la sphere vide," Bull. Acad. Sci. USSR, pp. 793-800, 1934.

[19] M. Costantini and P. A. Rosen, "A generalized phase unwrapping approach for sparse data," in Proc. IGARSS, vol. 1, Hamburg, Germany, June 1999, pp. 267-269.

[20] W. Xu and I. Cumming, "A region-growing algortihm for InSAR phase unwrapping,” IEEE Trans. Geosci. Remote Sensing, vol. 37, Jan. 1999.

[21] R. Hanssen, Atmospheric Heterogeneities in ERS Tandem SAR Interferometry. Delft, The Netherlands: Delft Univ. Press, 1998.

[22] — Radar Interferometry. Amsterdam, The Netherlands: Kluwer, 2001

[23] D. Massonet and K. L. Feigl, "Radar interferometry and its applications to changes in Earth's surface," Rev. Geophys., no. 36, pp. 441-500, 1998.

[24] M. D. Pritt and J. S. Shipman, "Least-Squares two-dimensional phase unwrapping using FFT's," IEEE Trans. Geosci. Remote Sensing, vol. 32, pp. 706-708, May 1994.

[25] A. V. Oppenheim and R. W. Schafer, Discrete-Time Signal Processing, ser. Signal Processing Series. Upper Saddle River, NJ: Prentice-Hall, 1989. 


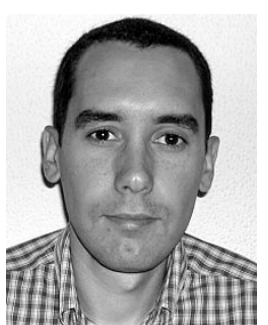

Oscar Mora (M'98) received the Electrical Engineer Degree from the Escola Tècnica Superior d'Enginyeria de Telecomunicació de Barcelona (ETSETB), Universitat Politècnica de Catalunya (UPC), Barcelona, Spain, in 1997.

He joined the Active Remote Sensing Group in 1999 in the frame of Ph.D. work in the field of SAR differential interferometry. From September to December 2001, he was with the Istituto per il Rilevamento Elettromagnetico dell'Ambiente (IREA), Naples, Italy, in the frame of his Ph.D. work. His research is based on the study and development of new orbital SAR differential interferometric techniques for the monitoring of terrain surface displacements, such as the ones produced by volcanoes, earthquakes, or human activities. Recently, he joined the Institut Cartogràfic de Catalunya (ICC) Barcelona, Spain, for continuing his research activities in the field of SAR interferometry.

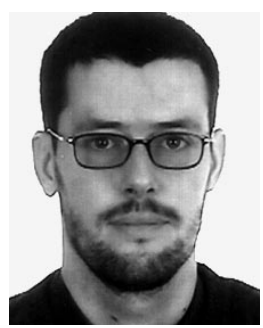

Jordi J. Mallorquí (S'90-M'96) was born in Tarragona, Spain, in 1966. He received the Ingeniero degree in telecommunication engineering and the Doctor Ingeniero degree in telecommunications engineering, in 1990 and 1995, respectively, both from the Universitat Politècnica de Catalunya (UPC), Barcelona, Spain.

In 1991, he joined the Department of Signal Theory and Communications as a Ph.D. student. In 1993, he became an Assistant Professor, and since 1997, he has been an Associate Professor at the Telecommunications Engineering School of UPC. His teaching activity involves microwaves, radio navigation, and remote sensing. He spent a sabbatical year at the Jet Propulsion Laboratory, Pasadena, CA, in 1999, working on interferometric airborne SAR calibration algorithms. He is currently working on the application of SAR interferometry to terrain subsidence monitoring with orbital and airborne data, vessel detection and classification from SAR images, and three-dimensional electromagnetic simulation of SAR systems. He has published more than 60 papers on microwave tomography, electromagnetic numerical simulation, SAR processing, interferometry, and differential interferometry.

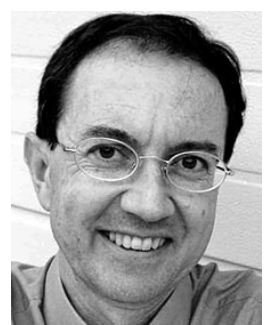

Antoni Broquetas (S'84-M'90) was born in Barcelona, Spain, in 1959. He received the Ingeniero degree in telecommunication engineering and the Doctor Ingeniero degree, in 1985 and 1989, respectively, both from the Universitat Politècnica de Catalunya (UPC), Barcelona, Spain.

In 1986, he was a Research Assistant with Portsmouth Polytechnic, Porstmouth, U.K., where he was involved in propagation studies. In 1987, he joined the Department of Signal Theory and Communications, School of Telecommunication Engineering, UPC. He was Subdirector of Research at the Institute of Geomatics, Barcelona, Spain. He is currently a Full Professor in UPC, where he is involved in research on radar imaging and remote sensing. He has published more than 150 papers on microwave tomography, radar, ISAR and SAR systems, SAR processing, and interferometry. 